\title{
Instrumentos psicológicos utilizados em seleção profissional
}

\author{
Silvia Leite de Godoy ${ }^{\star}$ \\ Ana Paula Porto Noronha ${ }^{\star \star}$
}

\section{Resumo}

Considerando a importância que os instrumentos de avaliação psicológica possuem nos contextos profissionais do psicólogo, entre os quais as organizações, o presente estudo teve como objetivos: a) identificar os instrumentos mais utilizados em seleção profissional; e b) identificar as técnicas mais empregadas nos processos seletivos. Participaram como sujeitos desta pesquisa 15 estudantes último anistas do curso de Psicologia de uma instituição de ensino superior particular do interior paulista e 15 psicólogos recrutadores e selecionadores da região de Campinas. O material utilizado se constituiu de um questionário enviado aos profissionais por e-mail e distribuído aos estudantes na universidade. Os resultados mostraram que os instrumentos psicológicos mais utilizados nas seleções foram Wartegg, Atenção Concentrada, Teste de Inteligência Não Verbal G36, Bateria CEPA e Palográfico e as técnicas mais empregadas foram entrevistas individuais, dinâmicas de grupo e testes psicológicos. Sugere-se novos estudos na área como fomento para ações seguras relacionadas à avaliação psicológica.

Palavras-chave: Avaliação psicológica. Instrumentos psicológicos. Proces$s$

seletivo.

\section{Psychological instruments of recruiting process}

\begin{abstract}
Considering the importance of psychological assessment instruments in professional contexts of the psychologist (in organizations among others), the present study has the purpose of identifying not only the main useful strategies of recruiting people, but also the most innovative practices in the recruiting process. A group of 15 graduate students, in their last year of the Psychology Course at a private university in São Paulo's countryside, and 15 psychologists, who work as

^ Psicóloga, formada pela Universidade São Francisco.

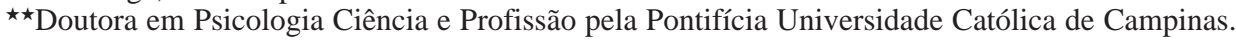
Docente e coordenadora do Programa de Pós-Graduação em Psicologia da Universidade São Francisco. End.: Rua Alexandre Rodrigues Barbosa, 45 - Centro Itatiba - SP C.E.P.: 13.251-900.

E-mail: ananoronha@saofrancisco.edu.br
\end{abstract}


recruiters in the city of Campinas, participated in this research. A questionnaire was emailed to professionals and handled out to the university students. The results showed that the Wartegg, Concentrated Attention, NonVerbal Intelligence Test G36, "Bateria CEPA" and "Palográfico" were the instruments most frequently used in the recruiting process, and the most common techniques were individuals interviews, group dynamic and psychological tests. New studies in this field are recommended in order to promote more reliable actions in psychological assessment.

Keywords: Psychological assessment. Psychological instruments. Recruiting process.

\section{Avaliação PSicológica: Conceito, uSo E FORMaÇão PROFISSIONAL}

Avaliação psicológica é um processo de coleta de dados, cuja realização inclui métodos e técnicas de investigação, dentre eles os testes psicológicos, que, por sua vez, são instrumentos exclusivos do psicólogo. Ao lado disso, esses instrumentos são úteis à medida que, quando utilizados adequadamente, podem oferecer informações importantes sobre os testandos.

Embora a avaliação psicológica seja reconhecidamente uma atividade exclusiva da categoria profissional, há que se considerar que ela é muito controvertida, sobretudo quando sua realização inclui o uso de testes. Enquanto alguns profissionais consideram os instrumentos como indispensáveis outros são absolutamente contrários, considerando-os como "instrumentos de poder" cuja única função é rotular os indivíduos. A crítica pode ser atribuída principalmente ao uso inadequado e incorreto dos testes por alguns profissionais, cuja conseqüência tende a ser prejudicial às pessoas avaliadas, além dos questionamentos pertinentes à falta de cientificidade dos próprios instrumentos (ALVES; ALCHIERI; MARQUES, 2002).

Aliado a isso, a formação em avaliação tem sido muito questionada e, nesse sentido, vale destacar que ela apresenta uma série de desafios no que se refere às constantes transformações com as quais a Psicologia se defronta neste novo século (BASTOS, 2002). A representação dos principais fundamentos da medida psicológica, a elaboração e a construção de novos testes, a necessidade de representar um conjunto de postulados e novas teorias, faz do ensino de avaliação psicológica, um interjogo constante entre conteúdos novos e antigos.

No estudo de Almeida, Prieto, Muñiz e Bartram (1998) foi constatado que existe uma clara relação entre problemas nos instrumentos psicológicos e problemas na formação profissional do psicólogo que constrói e que usa os referidos instrumentos. Ainda dando margem a esta questão, Noronha (2002) comenta que a formação de um psicólogo em cinco anos de universidade não é suficiente para aprimorá-lo em todas as áreas de conhecimento, embora devesse sê-lo. Autores concordam que se faz necessário modificar a formação, já que a Psicologia é uma 
profissão em transformação e isto implica novas atitudes em relação ao conhecimento, ao exercício da profissão e ao processo formador (DURAN, 1994; HUTZ; BANDEIRA, 2003; NORONHA; ALCHIERI, 2003).

Atualmente, os testes psicológicos têm recebido muitas críticas que variam desde a fundamentação teórica, passando pela ausência de estudos recentes e de adaptações para diferentes realidades, até o alto custo de determinados materiais (NORONHA, 1999; PRIETO; MUÑIZ, 2000; OLIVEIRA; NORONHA; DANTAS, 2005; NORONHA). Outros questionamentos dizem respeito a sua utilidade prática, tendo em vista que alguns autores não concordam com as propriedades que os próprios psicólogos atribuem ao instrumento padronizado.

Figueiredo e Pinheiro (1998) apontam que no Brasil a maior crítica feita aos testes psicológicos, à época do estudo, refere-se à falta de adaptações, uma vez que a maioria dos instrumentos disponíveis no mercado brasileiro é originária de outro país. Mesmo quando elaborados no Brasil, os testes são raramente submetidos a uma adaptação rigorosa, e as normas não são habitualmente atualizadas e revisadas após as publicações originais. Nos últimos anos, no entanto, não parece ser este o problema central dos instrumentos de medida, já que a pesquisa de Noronha, Primi e Alchieri (no prelo) revela que mais da metade dos instrumentos comercializados no país é de produção nacional. Em contrapartida, o estudo revela que aproximadamente metade dos testes não possui estudos de validade.

Embora as qualidades técnicas venham-se aperfeiçoando, apenas a formação do psicólogo aliada à competência dele, possibilitará uma compreensão mais ampla e contextualizada do processo de avaliação, permitindo uma interpretação mais adequada dos resultados. Trata-se, portanto, de aprimorar a formação do psicólogo em avaliação psicológica, incluindo no currículo dos cursos de Psicologia temas e conteúdos que reflitam ou fundamentem tal aprimoramento, permitindo, assim, que o psicólogo seja capaz de avaliar a qualidade dos instrumentos que utiliza e que saiba fazer uso adequado deles (NORONHA et. al., 2003)

O estatuto da avaliação psicológica, de acordo com Azevedo e outros (1996), tem grangeado uma melhor reputação no seio da investigação e da prática psicológica, nomeadamente por parte dos profissionais que lidam com recursos humanos em geral ou ainda dos órgãos que necessitam de avaliações periódicas e sistemáticas de seus projetos ou programas. Os autores mencionam que, comparativamente à situação estritamente clínica e individual de uso tradicional dos testes, atualmente se enfatiza mais uma avaliação dirigida aos contextos habituais de desempenho, como a apreciação da eficácia dos programas de intervenção, metodologias de trabalho ou condições de realização. Entretanto, são essas necessidades mais recentes que estão contribuindo para o crescimento do campo de atuação da avaliação psicológica na área de recursos humanos, mais do que o diagnóstico psicológico feito na avaliação psicológica tradicional.

Alchieri (1999) afirma que existem procedimentos na avaliação com regras e situações bem definidas que, seguidos fielmente, permitem a qualquer psicólogo obter o mesmo resultado que outro profissional. Outros procedimentos, por sua 
vez, exigem a intervenção interpretativa do examinador, tais como, julgar a adequação ou a categoria de uma determinada resposta, segundo modelos existentes e que podem exigir, também, a apreciação das condições do exame e julgamento de fatores externos, facilitadores ou não das tarefas propostas. Ainda no mesmo estudo, o autor alerta para a importância de os profissionais usuários de instrumentos psicológicos terem condições de entender os usos e as limitações dos testes que utilizam, considerando que eles são construídos para avaliar certos eventos, ou procedimentos, ou atributos, numa determinada população e sob circunstâncias restritas; de tal sorte que um único teste pode apresentar vários índices de validade, desde a inexistência dela até a sua mais alta expressão, sem que sua indicação possa estar assegurada para toda e qualquer atividade (APA; AERA; NCME, 1999).

Com essas considerações a respeito dos testes psicológicos, espera-se que os atuais serviços de avaliação psicológica tenham como meta redefinir os objetivos de trabalho, buscando a atualização de métodos e procedimentos empregados, bem como a escolha mais criteriosa de instrumentos psicológicos atualizados e em melhores condições técnicas, a fim de satisfazerem os requisitos da avaliação. Este trabalho poderá ser realizado por meio de parcerias entre editoras, órgãos e associações de classe, universidades e psicólogos. Mediante essa sistemática, é possível além de compor resultados mais imediatos, iniciar os estudos com a definição de uma política de ações, cooperando com diversos estados, e suas instituições científicas na área.

Alchieri (2003) sugere como propostas de ações para avaliação psicológica, a formação de profissionais mais capacitados, a atualização dos instrumentos e técnicas, investigações sistemáticas quanto à eficácia dos instrumentos disponíveis, planejamento e desenvolvimento de novos e mais específicos instrumentos, e informatização quanto à avaliação e aplicação.

Diante da importância da avaliação psicológica na prática profissional do psicólogo em qualquer contexto de trabalho, ressalta-se a necessidade de estudos e pesquisas na área, para que pesquisadores e psicólogos encontrem o devido estado de excelência, e possam exercer a profissão com dignidade, buscando o benefício da população.

\section{Seleção Profissional: Avanços e desafios}

Cada vez mais, os desafios de uma sociedade globalizada invadem a população brasileira levando-a a discutir, a refletir e a rever todos os conceitos em busca de novos paradigmas que possibilitem a reconstrução do pensar e do agir. Não é possível, nas organizações atuais, e nas vidas particulares, manter os mesmos padrões de relações e de resultados sem repensar os diversos contextos dos ambientes organizacionais ou sociais.

Nenhuma área da Psicologia passou por transformações tão profundas, durante o último século, como a que se dedica ao setor de recursos humanos, conforme afirma Castelar (2001). Afetado, de um lado, pela automação e pela crise eco- 
nômica que mudaram o perfil do emprego e, de outro, pelos novos métodos administrativos, por exemplo, a terceirização, o setor de recursos humanos vive uma mutação sem precedentes. Como resultado, o psicólogo que optou por essa especialidade acabou perdendo espaço e vive hoje uma crise de identidade.

Historicamente, para Cansian (2002), a Psicologia entrou nas empresas pela porta da seleção e avaliação de pessoal. O desenvolvimento de quaisquer atividades dentro ou fora das organizações depende - e muito - das condições das empresas, e, principalmente, do profissional que as realiza. O mercado, extremamente competitivo, exige cada vez mais competência técnica e interpessoal, de tal modo que tanto as condições pessoais (crenças, valores, postura, capacidade para colocar idéias) quanto as técnicas (formação acadêmica, cursos de especialização, domínio de tecnologias) auxiliarão a determinar o espaço de trabalho do profissional e a credibilidade de suas propostas.

De acordo com Schimdt e Hunter (1998) os psicólogos vêm-se dando conta de que os testes têm um papel importante no processo de seleção, à medida que os aspectos atualmente mais valorizados quebram paradigmas que sustentavam o processo seletivo ao longo de toda a história da humanidade, cujas características pessoais tornaram-se mais importantes que sua folha corrida de realizações. Houve uma época em que o currículo a ser apresentado em uma entrevista de seleção profissional era uma das principais preocupações do candidato. Hoje, este material é apenas utilizado como roteiro para que o entrevistador explore as questões que lhe interessam e que se relacionam com as características exigidas. O que o mercado está buscando é o profissional que desenvolva uma inesgotável capacidade de adaptação a toda gama de situações que possa vivenciar, que consiga enxergar no novo a sua motivação para vencer, que veja obstáculos como desafios a serem transpostos, e não como empecilhos à sua trajetória.

Benucci (2003) afirma que a maioria dos currículos que chegam às empresas não expressam detalhadamente a vida profissional, acadêmica e social do candidato, tornando-se necessário fazer uma co-relação entre os dados apresentados no currículo com a comunicação verbal e não-verbal do entrevistado. Dessa forma, não é apenas a entrevista que conta para o preenchimento de uma vaga: as dinâmicas de grupo, os testes psicológicos, entre outros, são instrumentos importantes para procurar um índice de acerto na contratação do candidato em relação às expectativas e necessidades do contratante.

Pereira, Primi e Cobêro (2003) referindo-se especialmente aos testes psicológicos, afirmam ser necessário atualizá-los, e que os profissionais devem ficar atentos às qualidades psicométricas dos instrumentos utilizados, sobretudo no que se refere à validade preditiva. Afirmam ainda, que os testes psicológicos são instrumentos de medida cientificamente desenvolvidos a partir de um longo investimento que se caracteriza por sua rigidez metodológica e ampla fundamentação teórica. Atualmente, a prática psicológica na seleção profissional está comprometida devido ao mau uso dos instrumentos de avaliação. O que pode vir a colocar em risco a qualidade de um processo seletivo é a incompetência do selecionador 
no uso da técnica, e não a técnica em si. É necessário admitir que há variáveis próprias do sujeito que podem facilitar ou dificultar sua atuação no trabalho, de forma que se busca numa seleção não apenas a super qualificação ou máxima experiência, mas a maior compatibilidade possível entre as características do sujeito e as exigências do cargo que pretende ocupar.

Independentemente da ferramenta que se utilize na seleção profissional, o essencial é manter o ciclo de satisfação de ambas as partes, procurando incluir um profissional qualificado de acordo com as necessidades da empresa. O profissional feliz com a sua ocupação apresenta uma tendência maior para atuar com assertividade e eficácia, e, conseqüentemente, agregará mais valor para a empresa (CANSIAN, 2002).

Inúmeras são as ferramentas usadas num processo de seleção, dentre elas, Cansian (2002), destaca a aplicação de testes que ajudam o selecionador a traçar um perfil mais preciso do candidato. Entretanto, assim como entrevistas e dinâmicas de grupo, os testes são instrumentos importantes e necessários nos processos seletivos. Devem fazer parte de um conjunto de informações acerca dos candidatos. Não foram elaborados para rotular pessoas nem impedir contratações. Foram, e são, inventados para dar aos selecionadores informações que dificilmente poderiam obter de alguma outra forma. Além disso, não são definidos como "certos" e "errados". O conjunto de informações colhidas mostra se o profissional atinge o perfil necessário à empresa, se possui potencial ou não para desempenhar as atividades propostas. Um outro aspecto apontado pelo autor é a substituição gradativa dos testes psicológicos por instrumentos subjetivos de avaliação, como as dinâmicas de grupo ou as entrevistas, ferramentas com as quais qualquer profissional pode, atualmente, trabalhar em seleção de recursos humanos.

Todo este cenário, segundo Pereira, Primi e Cobêro (2003), faz parte das atribuições do psicólogo, que tem de se preparar para refletir a respeito dos profundas mudanças que estão ocorrendo, atentando-se para os novos apelos que surgem. Esse é um dos aspectos que diferencia alguns profissionais e os fazem conquistar e manter cada vez mais seu espaço no mercado de trabalho. Quem não tiver consciência e não captar as dimensões da mudança, não estará apto para lidar com as bases de uma nova ordem mundial. Erros e acertos são o que mais têm acontecido no mundo das empresas que passaram e que passam por transformações.

Tendo em vista a necessidade de estudos atuais sobre essa área de conhecimento, bem como de pessoas capazes e motivadas para assegurar a competitividade da empresa, o presente trabalho teve como objetivos identificar os instrumentos mais utilizados/conhecidos em seleção profissional e identificar as técnicas mais empregadas nos processos seletivos.

\section{Método}

O método foi dividido nas etapas que se seguem. 


\section{Participantes}

Participaram como sujeitos da pesquisa 30 indivíduos entre estudantes de psicologia e psicólogos, sendo 97\% (F=29) do sexo feminino e $3 \%(\mathrm{~F}=1)$ do sexo masculino, divididos em dois grupos, a saber:

Grupo I: 15 profissionais recrutadores e selecionadores da região de Campinas. As idades variaram entre 23 e 50 anos, sendo a média de 34 anos. Os sujeitos são formados em média há nove anos, dos quais $33 \%(\mathrm{~F}=5)$ concluíram sua graduação na Pontifícia Universidade Católica de Campinas, 20\% ( $\mathrm{F}=3$ ) na Universidade Metodista de Piracicaba, 20\% ( $\mathrm{F}=3$ ) na Universidade São Francisco, 7\% ( $\mathrm{F}=1$ ) na Universidade Federal do Ceará, 7\% ( $\mathrm{F}=1)$ na Universidade de Santo Amaro, 7\% ( $\mathrm{F}=1)$ na Universidade Metodista de São Paulo e 7\% ( $\mathrm{F}=1)$ na Universidade Faria Brito. Em relação à titulação máxima verificou-se que: $7 \%(\mathrm{~F}=1)$ são mestres, 27\% ( $\mathrm{F}=4)$ são especialistas e 67\% ( $\mathrm{F}=10)$ psicólogos, dos quais 40\% ( $\mathrm{F}=6)$ atuam na abordagem comportamental, 33\% ( $\mathrm{F}=5)$ na abordagem humanista e $27 \%$ $(\mathrm{F}=4)$ na abordagem psicanalítica. Apenas $14 \%(\mathrm{~F}=2)$ dos profissionais exercem atividades paralelas à atuação organizacional: e $7 \%(\mathrm{~F}=1)$ atuam em consultório particular e $7 \%(\mathrm{~F}=1)$ em instituição.

Grupo II: 15 estudantes último anistas do curso de Psicologia, de uma instituição de ensino superior particular do interior paulista, com média de idade de 27 anos, variando entre 23 e 36 anos. No que se refere à atuação vimos que: $40 \%$ $(\mathrm{F}=6)$ dos estudantes atuam na abordagem humanista, 33\% ( $\mathrm{F}=5)$ na abordagem comportamental e $27 \%(\mathrm{~F}=4)$ na abordagem psicanalítica. Todos os participantes atuam em organizações exercendo atividades de recrutamento e seleção.

\section{Material}

O material utilizado para a coleta de dados constituiu-se de um questionário contendo questões fechadas, divididas em três partes, a saber:

1.Dados de identificação que se referem à formação, tais como, tempo de graduação, instituição e titulação, bem como atuação profissional no que diz respeito a outras atividades exercidas paralelamente ao trabalho organizacional, como, por exemplo, o tempo que se dedica a ela e respectiva abordagem trabalhada;

2.Estratégias e instrumentos utilizados na seleção profissional como entrevistas, dinâmicas, inventários, escalas e testes;

3.Instrumentos psicológicos específicos mais utilizados e conhecidos na prática profissional.

\section{Procedimento}

Os instrumentos foram aplicados com a devida autorização dos sujeitos. Para os profissionais formados, os questionários foram enviados por e-mail e a 
orientação foi realizada coletivamente em uma reunião de recrutadores e selecionadores em horário previamente cedido pelo grupo. Sua devolução variou de dois a dez dias. Para a coleta de dados dos alunos, os questionários foram distribuídos aleatoriamente na universidade e devolvidos na semana seguinte.

\section{Resultados}

De acordo com o primeiro objetivo do trabalho, procurou-se investigar quais os instrumentos mais utilizados em seleção profissional pelos psicólogos e pelos estudantes do curso de Psicologia. As respostas obtidas foram agrupadas em cinco Tabelas: a primeira refere-se aos instrumentos psicológicos mais conhecidos pelos psicólogos, a segunda aos instrumentos mais utilizados por esses profissionais, a terceira apresenta os instrumentos psicológicos mais conhecidos pelos estudantes de Psicologia, a quarta os instrumentos mais utilizados por esses indivíduos e a quinta os instrumentos que não receberam citação pelos psicólogos e estudantes.

$\mathrm{Na}$ Tabela 1 foram enumerados 94 instrumentos psicológicos dos mais conhecidos para os menos conhecidos, ou seja, foram organizados em ordem decrescente de acordo com o número de profissionais que os conhecem. Dos 94 instrumentos apresentados, 12 foram destacados como mais conhecidos pela maioria dos psicólogos, sendo que de 15 psicólogos, 11 conhecem o Teste de Inteligência

Tabela 1: Instrumentos psicológicos mais conhecidos pelos psicólogos

\begin{tabular}{|r|l|c|}
\hline \multicolumn{2}{|c|}{ Instrumentos Psicológicos } & Conhecidos \\
\hline $\mathbf{1}$ & Teste de Inteligência Não Verbal G36 & 11 \\
\hline $\mathbf{2}$ & O desenho da figura humana & 11 \\
\hline $\mathbf{3}$ & Teste de Rorschach & 10 \\
\hline $\mathbf{4}$ & Teste Palográfico & 10 \\
\hline $\mathbf{5}$ & Bender Infantil & 9 \\
\hline $\mathbf{6}$ & Bateria CEPA & 9 \\
\hline $\mathbf{7}$ & Teste Psicodiagnóstico Miocinético-PMK & 9 \\
\hline $\mathbf{8}$ & Atenção Concentrada & 8 \\
\hline $\mathbf{9}$ & Teste de Inteligência Não Verbal G38 & 8 \\
\hline $\mathbf{1 0}$ & WISC III & 8 \\
\hline $\mathbf{1 1}$ & Dominós D-48 & 7 \\
\hline $\mathbf{1 2}$ & Teste Wartegg & 7 \\
\hline $\mathbf{1 3}$ & Inventário de Atitudes de Trabalho-IAT & 6 \\
\hline $\mathbf{1 4}$ & Escala de Maturidade Mental Columbia & 5 \\
\hline $\mathbf{1 5}$ & O Teste das Pirâmides das Cores & 5 \\
\hline $\mathbf{1 6}$ & O Teste Gestaltico Bender para Crianças & 5 \\
\hline $\mathbf{1 7}$ & Teste Raven Operações Lógicas - RTLO & 5 \\
\hline $\mathbf{1 8}$ & Inventário de Interesses Profissionais & \\
\hline
\end{tabular}




\begin{tabular}{|c|c|c|}
\hline & Instrumentos Psicológicos & Conhecidos \\
\hline 19 & Matrizes Progressivas - escala geral & 4 \\
\hline 20 & QVI Questionário Vocacional Interesses & 4 \\
\hline 21 & Teste de Apercepção Temática TAT & 4 \\
\hline 22 & Teste de Aptidões Específicas DAT & 4 \\
\hline 23 & Teste Zulliger & 4 \\
\hline 24 & D2-Teste de Atenção Concentrada & 4 \\
\hline 25 & Teste das Pirâmides das Cores 24M-Pfister & 4 \\
\hline 26 & $\begin{array}{l}\text { Inventário de Sintomas de Stress para } \\
\text { Adultos Lipp }\end{array}$ & 3 \\
\hline 27 & Como Chefiar? & 3 \\
\hline 28 & Escala de Inteligência Wechsler Crianças & 3 \\
\hline 29 & Inventário Fatorial de Personalidade & 3 \\
\hline 30 & Matrizes Progressivas - escala avançada & 3 \\
\hline 31 & INV & 3 \\
\hline 32 & QUATI Questionário Avaliação Tipológica & 3 \\
\hline 33 & Teste de Apercepção Infantil CAT-A & 3 \\
\hline 34 & Teste D-70 & 3 \\
\hline 35 & $\begin{array}{l}\text { ACRE- Teste de Atenção Concentrada, } \\
\text { Rapidez e Exatidão }\end{array}$ & 3 \\
\hline 36 & Atenção Concentrada 15 & 2 \\
\hline 37 & Escala de Personalidade de Comrey & 2 \\
\hline 38 & Escala de Maturidade Escolha Profissional & 2 \\
\hline 39 & Supl. Teste Apercepção Infantil CAT-S & 2 \\
\hline 40 & Teste das Cores & 2 \\
\hline 41 & Teste das Pirâmides Coloridas & 2 \\
\hline 42 & Teste de Apercepção Infantil CAT-H & 2 \\
\hline 43 & Teste dos Relógios & 2 \\
\hline 44 & Inventário de Interesses Angelini e Thustone & 2 \\
\hline 45 & $\begin{array}{l}\text { Inventário e Auto-análise dos Interesses } \\
\text { Prof. IAIP }\end{array}$ & 2 \\
\hline 46 & R-2 Teste não verbal de inteligência & 2 \\
\hline 47 & Bateria Burocrática VIG & 1 \\
\hline 48 & Bateria de Testes de Aptidão BTAG & 1 \\
\hline 49 & Bateria TSP & 1 \\
\hline 50 & Cubos de Kohs & 1 \\
\hline 51 & Destreza Digital & 1 \\
\hline 52 & Dezesseis PF & 1 \\
\hline 53 & Diagnóstico Organizacional & 1 \\
\hline 54 & Escala de Preconceito Profissional-EPP & 1 \\
\hline 55 & Escala de Sociabilidade e Emotividade-ESE & 1 \\
\hline 56 & Escala de Stress Infantil-ESI & 1 \\
\hline
\end{tabular}




\begin{tabular}{|l|l|c|}
\hline \multicolumn{2}{|c|}{ Instrumentos Psicológicos } & Conhecidos \\
\hline $\mathbf{5 7}$ & Escala Reduzida de Autoconceito - ERA & 1 \\
\hline $\mathbf{5 8}$ & Escore de Deteriorização Desenho Pessoa & 1 \\
\hline $\mathbf{5 9}$ & $\begin{array}{l}\text { Escala de Transtorno de Déficit de } \\
\text { Atenção/Hiperatividade }\end{array}$ & 1 \\
\hline $\mathbf{6 0}$ & Figuras Complexas de Rey & 1 \\
\hline $\mathbf{6 1}$ & Inventário de Administração de Tempo- & 1 \\
\hline $\mathbf{6 2}$ & Inventário de Ansiedade IDATE & 1 \\
\hline $\mathbf{6 3}$ & Inventário de Ansiedade IDATE-C & 1 \\
\hline $\mathbf{6 4}$ & IAR Instrum. Aval. Repert. Básico Alfab. & 1 \\
\hline $\mathbf{6 5}$ & Kuder Invent. De Interesses Vocacional & 1 \\
\hline $\mathbf{6 6}$ & LIP Levantamento de Interesses Profission. & 1 \\
\hline $\mathbf{6 7}$ & Liderança e Poder & 1 \\
\hline $\mathbf{6 8}$ & Lista de Problemas Pessoais adultos & 1 \\
\hline $\mathbf{6 9}$ & Lista de Problemas Pessoais adolescentes & 1 \\
\hline $\mathbf{7 0}$ & Matrizes Progressivas Coloridas & 1 \\
\hline $\mathbf{7 1}$ & Medida de Fluência Verbal-MFV & 1 \\
\hline $\mathbf{7 2}$ & Memória-R & 1 \\
\hline $\mathbf{7 3}$ & O Desenvolv. Comp. Criança Primeiro Ano & 1 \\
\hline $\mathbf{7 4}$ & Panorama de Atitudes dos Pais-PAP & 1 \\
\hline $\mathbf{9 7}$ & Programação Hábitos e Desempenhos-PHD & 1 \\
\hline $\mathbf{7 6}$ & Prova de Nível Mental & 1 \\
\hline $\mathbf{7 7}$ & Questionário Confidencial & 1 \\
\hline $\mathbf{7 8}$ & Reprodução de Figuras & 1 \\
\hline $\mathbf{7 9}$ & Sondagem de Habilidade & 1 \\
\hline $\mathbf{8 0}$ & Teste de Atenção Difusa & 1 \\
\hline $\mathbf{8 1}$ & Teste de Capacidades Intelectuais TCI & 1 \\
\hline $\mathbf{8 2}$ & Teste de Estruturas Vocacionais-TEV & 1 \\
\hline $\mathbf{8 3}$ & Teste de Liderança Situacional-TLS & 1 \\
\hline $\mathbf{8 4}$ & Teste Raciocínio Lógico-Numérico & 1 \\
\hline $\mathbf{8 5}$ & Teste de Sondagem Intelectual & 1 \\
\hline $\mathbf{8 6}$ & Teste Diagn. Habilidade Pré-escolar-DHP & 1 \\
\hline $\mathbf{8 7}$ & Teste de Retenção Visual - BENTOntil ESI & 1 \\
\hline
\end{tabular}


Não Verbal G36 e o Desenho da Figura Humana, 10 conhecem o Rorschach e o Teste Palográfico, 9 conhecem o Bender Infantil, a Bateria CEPA e o Teste Psicodiagnóstico Miocinético (PMK), 8 conhecem Atenção Concentrada, o Teste

Tabela 2: Instrumentos psicológicos mais utilizados pelos psicólogos

\begin{tabular}{|c|c|c|}
\hline \multicolumn{2}{|r|}{ Instrumentos Psicológicos } & Utilizados \\
\hline 1 & Atenção Concentrada & 7 \\
\hline 2 & Teste Wartegg & 6 \\
\hline 3 & Atenção Concentrada 15 & 4 \\
\hline 4 & Teste de Inteligência Não Verbal G36 & 4 \\
\hline 5 & Teste Palográfico & 4 \\
\hline 6 & Teste de Inteligência Não Verbal G38 & 3 \\
\hline 7 & Dominós D-48 & 2 \\
\hline 8 & Bateria CEPA & 2 \\
\hline 9 & Teste Não Verbal de Inteligência R-1 & 2 \\
\hline 10 & Escala de Maturidade Mental Columbia & 1 \\
\hline 11 & Inventário de Interesses Profissionais & 1 \\
\hline 12 & LIP Levantamento de Interesses Profission. & 1 \\
\hline 13 & Matrizes Progressivas - escala avançada & 1 \\
\hline 14 & Matrizes Progressivas - escala geral & 1 \\
\hline 15 & O desenho da figura humana & 1 \\
\hline 16 & INV & 1 \\
\hline 17 & O Teste Gestaltico Bender para Crianças & 1 \\
\hline 18 & QUATI Questionário Avaliação Tipológica & 1 \\
\hline 19 & Teste das Pirâmides Coloridas & 1 \\
\hline 20 & Teste Raciocínio Lógico-Numérico & 1 \\
\hline 21 & Teste de Rorschach & 1 \\
\hline 22 & Teste D-70 & 1 \\
\hline 23 & Teste Raven Operações Lógicas - RTLO & 1 \\
\hline 24 & Teste Zulliger & 1 \\
\hline 25 & Teste das Pirâmides das Cores 24M-Pfister & 1 \\
\hline 26 & WISC III & 1 \\
\hline
\end{tabular}

de Inteligência Não Verbal G38 e o WISC III e 7 conhecem o D-48 e o Teste Wartegg.

O mesmo procedimento foi usado para a construção da Tabela 2, sendo enumerados 26 instrumentos psicológicos dos mais utilizados para os menos utilizados, organizados em ordem decrescente de acordo com o número de profissionais que os utilizam em sua prática. 
De 15 psicólogos que participaram desse estudo (Grupo I), sete utilizam Atenção Concentrada, seis utilizam o Teste Wartegg, quatro utilizam Atenção Concentrada 15, Teste de Inteligência Não Verbal G36 e Palográfico, três utilizam Teste de Inteligência Não Verbal G38, dois utilizam D-48, Bateria CEPA e Teste Não Verbal de Inteligência R-1 e pelo menos um dos profissionais utiliza Escala de Maturidade Mental Columbia, Inventário de Interesses Profissionais, LIP Levantamento de Interesses Profissionais, Matrizes Progressivas - escala avançada, Matrizes Progressivas - escala geral, Desenho da Figura Humana, INV, Teste Gestáltico Bender para Crianças, QUATI - Questionário Avaliação Tipológica, Teste das Pirâmides Coloridas, Teste Raciocínio Lógico - Numérico, Rorschach, D-70, RTLO, Zulliger, Testes das Pirâmides das Cores 24M - Pfister e WISC III.

Tabela 3: Instrumentos psicológicos mais conhecidos pelos estudantes de psicologia

\begin{tabular}{|c|c|c|}
\hline \multicolumn{2}{|r|}{ Instrumentos Psicológicos } & \multirow{2}{*}{$\begin{array}{c}\text { Conhecidos } \\
15\end{array}$} \\
\hline 1 & Teste Zulliger & \\
\hline 2 & Bender Infantil & 13 \\
\hline 3 & Escala de Maturidade Mental Columbia & 13 \\
\hline 4 & Teste de Apercepção Temática TAT & 13 \\
\hline 5 & Dezesseis PF & 12 \\
\hline 6 & O desenho da figura humana & 12 \\
\hline 7 & Invent. Multifásico Personalidade MMPI & 10 \\
\hline 8 & Teste das Fábulas & 10 \\
\hline 9 & Teste de Rorschach & 8 \\
\hline 10 & Inventário de Atitudes de Trabalho-IAT & 7 \\
\hline 11 & O Teste Gestaltico Bender para Crianças & 7 \\
\hline 12 & Teste de Apercepção Infantil CAT-A & 7 \\
\hline 13 & Teste de Apercepção Infantil CAT-H & 7 \\
\hline 14 & WISC III & 7 \\
\hline 15 & $\begin{array}{l}\text { Inventário de Sintomas de Stress para } \\
\text { Adultos Lipp }\end{array}$ & 6 \\
\hline 16 & BPR-5 & 5 \\
\hline 17 & Inventário Fatorial de Personalidade & 5 \\
\hline 18 & Supl.Teste Apercepção Infantil CAT-S & 5 \\
\hline 19 & Teste Palográfico & 5 \\
\hline 20 & Teste Psicodiagnóstico Miocinético-PMK & 5 \\
\hline 21 & Inventário de Habilidades Sociais & 5 \\
\hline 22 & Escala de Inteligência Wechsler Crianças & 4 \\
\hline 23 & Teste de Inteligência Não Verbal G38 & 4 \\
\hline 24 & $\begin{array}{l}\text { LIP Levantamento de Interesses } \\
\text { Profissionais }\end{array}$ & 4 \\
\hline 25 & D2-Teste de Atenção Concentrada & 4 \\
\hline
\end{tabular}




\begin{tabular}{|c|c|c|}
\hline & Instrumentos Psicológicos & Conhecidos \\
\hline 26 & Inventário de Interesses Angelini e Thustone & 4 \\
\hline 27 & Atenção Concentrada & 3 \\
\hline 28 & Teste Wartegg & 3 \\
\hline 29 & Atenção Concentrada 15 & 2 \\
\hline 30 & Bateria de Testes de Aptidão BTAG & 2 \\
\hline 31 & Como Chefiar? & 2 \\
\hline 32 & Diagnóstico Organizacional & 2 \\
\hline 33 & Escala de Maturidade Escolha Profissional & 2 \\
\hline 34 & $\begin{array}{l}\text { Escala de Transtorno de Déficit de Atenção / } \\
\text { Hiperatividade }\end{array}$ & 2 \\
\hline 35 & Teste de Inteligência Não Verbal G36 & 2 \\
\hline 36 & Inventário de Ansiedade IDATE & 2 \\
\hline 37 & Inventário de Interesses Profissionais & 2 \\
\hline 38 & Kuder Invent. de Interesses Vocacional & 2 \\
\hline 39 & Teste de Estruturas Vocacionais-TEV & 2 \\
\hline 40 & Teste D-70 & 2 \\
\hline 41 & $\begin{array}{l}\text { ACRE- Teste de Atenção Concentrada, } \\
\text { Rapidez e Exatidão }\end{array}$ & 2 \\
\hline 42 & Bateria Burocrática VIG & 1 \\
\hline 43 & Becasse Maturidade Escolar & 1 \\
\hline 44 & Diagnóstico Tipológico Organizacional & 1 \\
\hline 45 & Dominós D-48 & 1 \\
\hline 46 & Escala de Stress Infantil-ESI & 1 \\
\hline 47 & Inventário de Ansiedade IDATE-C & 1 \\
\hline 48 & Medida de Fluência Verbal-MFV & 1 \\
\hline 49 & O Teste das Pirâmides das Cores & 1 \\
\hline 50 & QUATI Questionário Avaliação Tipológica & 1 \\
\hline 51 & Questionário de Personalidade Dadahie & 1 \\
\hline 52 & Teste de Apercepção para Idosos & 1 \\
\hline 53 & Bateria CEPA & 1 \\
\hline 54 & Teste de Aptidões Específicas DAT & 1 \\
\hline 55 & Teste de Maturidade para Leitura - TML & 1 \\
\hline 56 & Teste Organização Percepto-Motora-TOP & 1 \\
\hline 57 & Teste dos Relógios & 1 \\
\hline 58 & Teste Metropolitano de Prontidão & 1 \\
\hline 59 & Teste de Prontidão para Leitura & 1 \\
\hline 60 & Teste Raven Operações Lógicas - RTLO & 1 \\
\hline 61 & MTB - série Both de testes manuais & 1 \\
\hline 62 & Escala de Défict de Atenção Hiperatividade & 1 \\
\hline 63 & TAA-Teste de Aptidão Acadêmica & 1 \\
\hline 64 & Escala de Stress Infantil ESI & 1 \\
\hline
\end{tabular}


Analisando os testes utilizados em relação aos conhecidos, observou-se que dos 94 instrumentos psicológicos conhecidos pelos psicólogos, apenas $28 \%$ ( $\mathrm{F}=26)$ são usados, o que demonstrou uma baixa utilização do instrumental quando comparado com os testes destacados como conhecidos citados na Tabela 2, presentes também na Tabela 1. Ainda no que se refere aos dados apresentados, causa estranheza a utilização de alguns instrumentos no contexto organizacional, tais como, Escala de Maturidade Mental Columbia ou, ainda, o WISC III, que se destinam a avaliar crianças ou adolescentes..

A Tabela 3 apresenta os instrumentos psicológicos mais conhecidos pelos estudantes de Psicologia. Foram enumerados 64 instrumentos dos mais conhecidos para os menos conhecidos, organizados em ordem decrescente de acordo com o número de estudantes que os conhecem. Dos 64 instrumentos apresentados, 14 foram destacados como conhecidos pela maioria dos sujeitos, dos quais de 15 estudantes, 15 conhecem Zulliger, o que representou 100\% da amostra, 13 conhecem Bender Infantil, Escala de Maturidade Mental Columbia e Teste de Apercepção Temática 12 conhecem Dezesseis PF e Desenho da Figura humana, 10 conhecem

Tabela 4: Instrumentos psicológicos mais utilizados pelos estudantes de psicologia

\begin{tabular}{|c|l|c|}
\hline \multicolumn{2}{|c|}{ Instrumentos Psicológicos } & Utilizados \\
\hline $\mathbf{1}$ & Teste Wartegg & 12 \\
\hline $\mathbf{2}$ & Atenção Concentrada & 8 \\
\hline $\mathbf{3}$ & Bateria CEPA & 5 \\
\hline $\mathbf{4}$ & Teste Palográfico & 5 \\
\hline $\mathbf{5}$ & Inventário de Atitudes de Trabalho-IAT & 4 \\
\hline $\mathbf{6}$ & Dezesseis PF & 3 \\
\hline $\mathbf{7}$ & Teste de Inteligência Não Verbal G36 & 3 \\
\hline $\mathbf{8}$ & LIP Levantamento de Interesses Profiss. & 2 \\
\hline $\mathbf{9}$ & Inventário de Sintomas de Stress para & 1 \\
\hline $\mathbf{1 0}$ & Ddultos Lipp & 1 \\
\hline $\mathbf{1 1}$ & O deseninós D-48 & 1 \\
\hline $\mathbf{1 2}$ & QUATI Questionário Avaliação Tipológica & 1 \\
\hline $\mathbf{1 3}$ & Teste de Aptidões Específicas DAT & 1 \\
\hline $\mathbf{1 4}$ & Teste D-70 & 1 \\
\hline
\end{tabular}

MMPI e Teste das Fábulas, oito conhecem Rorschach e sete conhecem Inventário de Atitudes de Trabalho (IAT), Teste Gestáltico Bender para Crianças, CAT-A Teste de Apercepção Infantil, CAT-H Teste de Apercepção Infantil e WISC III.

Os instrumentos psicológicos mais utilizados pelos estudantes de Psicologia são apresentados na Tabela 4, na qual foram enumerados 16 instrumentos, dos mais utilizados para os menos utilizados e organizados em ordem decrescente, de acordo com o número de estudantes que os utilizam na seleção profissional. 
Dos 15 indivíduos que participaram dessa pesquisa (Grupo II), 12 utilizam Teste Wartegg; oito utilizam Atenção Concentrada; cinco utilizam Bateria CEPA e Teste Palográfico; quatro utilizam- Inventários de Atitudes de Trabalho (IAT); três utilizam Dezesseis PF e Teste de Inteligência Não-Verbal G36; dois utilizamLevantamento de Interesses Profissionais (LIP) e um dos estudantes utiliza Inventário de Sintomas de Stress para Adultos Lipp, Dominós D-48, Desenho da Figura Humana, Questionário Avaliação Tipológica (QUATI), Teste de Aptidões Específicas e D-70. Os resultados encontrados estão em consonância com os de Pereira, Primi e Cobêro (2003), que estudam os instrumentos e técnicas mais utilizados em seleção de pessoal, além de verificar se os profissionais têm informações sobre as qualidades psicométricas deles.

Neste grupo, foi verificada uma baixa utilização dos testes psicológicos nos processos seletivos, pois, tomando como referência os instrumentos psicológicos

Tabela 5: Instrumentos psicológicos não-citados pelos psicólogos e estudantes

\begin{tabular}{|r|l|}
\hline & \multicolumn{1}{|c|}{ Instrumentos Psicológicos não citados } \\
\hline $\mathbf{1}$ & BBT \\
\hline $\mathbf{2}$ & Coleção Papel de Carta \\
\hline $\mathbf{3}$ & Cornell Index \\
\hline $\mathbf{4}$ & Inventário S.T.A.X.I \\
\hline $\mathbf{5}$ & Inventário Expectativas e Crenças-IECPA \\
\hline $\mathbf{6}$ & Inventário Ilustrado de Interesses-GEIST \\
\hline $\mathbf{7}$ & Mandala de Palavras \\
\hline $\mathbf{8}$ & Questionário do Adolescente R-4 \\
\hline $\mathbf{9}$ & Questionário Íntimo \\
\hline $\mathbf{1 0}$ & Teste Barcelona \\
\hline $\mathbf{1 1}$ & Teste Caracterológico \\
\hline $\mathbf{1 2}$ & Teste Coletivo Inteligência Adultos-CIA \\
\hline $\mathbf{1 3}$ & Teste de Agradabilidade Básica \\
\hline $\mathbf{1 4}$ & Teste de Catálogo Livros Bessa-Tramer \\
\hline $\mathbf{1 5}$ & Teste Compreensão Técnico-Mecânica \\
\hline $\mathbf{1 6}$ & Teste de Conceitos Básicos de Bohem \\
\hline $\mathbf{1 7}$ & Teste de Desempenho Escolar - TDE \\
\hline $\mathbf{1 8}$ & Teste de Frustração \\
\hline $\mathbf{1 9}$ & Teste Habilidade Trabalho Mental-HTM \\
\hline $\mathbf{2 0}$ & Teste Prontidão Emoc.Motoristas-TEPEM \\
\hline $\mathbf{2 1}$ & Teste Eqüicultural Inteligência \\
\hline $\mathbf{2 2}$ & Teste Desenho Silver-cognição e emoção \\
\hline $\mathbf{2 3}$ & Teste Edites de Inteligência - TEI \\
\hline $\mathbf{2 4}$ & Teste McQuarrie Relações Espaciais \\
\hline $\mathbf{2 5}$ & Teste Projetivo Ômega \\
\hline & \\
\hline
\end{tabular}




\begin{tabular}{|l|l|}
\hline & \multicolumn{1}{|c|}{ Instrumentos Psicológicos não citados } \\
\hline $\mathbf{2 6}$ & Teste Projetivo Sonoro \\
\hline $\mathbf{2 7}$ & Teste Prontidão Horizontes \\
\hline $\mathbf{2 8}$ & Becasse-Maturidade Escolar \\
\hline $\mathbf{2 9}$ & Becasse Atitudes Sócio-emocionais Crianças Pré-escolares \\
\hline $\mathbf{3 0}$ & BTN - Bateria de Testes Neuropcológicos \\
\hline $\mathbf{3 1}$ & Coordenação Bi-manual- Edites \\
\hline $\mathbf{3 2}$ & Escala de Avaliação do Comport. Infantil para Professor \\
\hline $\mathbf{3 3}$ & Lendo e Escrevendo \\
\hline $\mathbf{3 4}$ & MM- Teste as Minhas Mãos \\
\hline $\mathbf{3 5}$ & Psicônica- Programador- Previsor de Desempenho \\
\hline $\mathbf{3 6}$ & Questionário Desiderativo \\
\hline $\mathbf{3 7}$ & Teste das Dinâmicas Profissionais - TDP \\
\hline $\mathbf{3 8}$ & Teste de Aptidão Á Mecânica- TAM \\
\hline $\mathbf{3 9}$ & DTVP-2 Teste Evolutivo de Percepção Visual \\
\hline $\mathbf{4 0}$ & BFM 1 \\
\hline $\mathbf{4 1}$ & BFM 2 \\
\hline $\mathbf{4 2}$ & Escala Fatorial de Ajustamento Emocional/ Neuroticismo \\
\hline
\end{tabular}

utilizados em relação aos conhecidos, observou-se que dos 64 testes conhecidos pelos estudantes, apenas $22 \%$ ( $\mathrm{F}=14$ ) são utilizados, o que constatou que sua importância ainda é pouco reconhecida pelos profissionais que atuam na área de recrutamento e seleção. Em contrapartida, vale destacar que este grupo era composto por estudantes de psicologia, que, costumeiramente, possuem menos autonomia na escolha e determinação dos instrumentos usados nos processos.

A Tabela 5 apresenta os instrumentos que não foram citados pelos profissionais e estudantes de Psicologia. Verificou-se entre os dois grupos que $28 \%$ ( $F=42$ ) do total da amostra de 152 instrumentos psicológicos específicos não são conhecidos e utilizados, o que demonstra que apenas $72 \%$ dos testes são conhecidos e/ou utilizados por esses indivíduos.

Nas respostas dos psicólogos referente ao total da lista de instrumentos, 58 testes não receberam citação, o que significa que $39 \%$ não são conhecidos e utilizados. Em relação às respostas dos estudantes, observou-se que 88 instrumentos psicológicos não receberam citação, o que indica que 58\% dos testes apresentados não são conhecidos e utilizados por esses sujeitos.

Os resultados indicaram uma diferença entre os grupos, no que diz respeito aos instrumentos mais conhecidos e utilizados pelos profissionais e estudantes de Psicologia. Da relação apresentada de 152 instrumentos psicológicos, $62 \%$ ( $\mathrm{F}=94)$ são conhecidos pelos psicólogos (Grupo I) e 18\% ( F=26) são utilizados na prática profissional. Da mesma lista de instrumentos, $43 \%$ ( $\mathrm{F}=64)$ são conhecidos pelos estudantes (Grupo II) e apenas 10\% ( $\mathrm{F}=14)$ são utilizados. 
Em relação ao segundo objetivo do estudo, ou seja, identificar as técnicas mais empregadas nos processos seletivos, houve uma análise da freqüência de respostas das estratégias mais utilizadas em seleção profissional. As técnicas empregadas nesta análise foram entrevistas individuais, entrevistas coletivas, dinâ-

Tabela 6: Técnicas utilizadas em seleção profissional pelos psicólogos

\begin{tabular}{|l|c|c|}
\hline \multirow{2}{*}{ Técnicas de Seleção Profissional } & \multicolumn{2}{|c|}{ Técnicas Utilizadas } \\
\cline { 2 - 3 } & F & \% \\
\hline Entrevistas Individuais & 15 & 100 \\
\hline Dinâmicas de Grupo & 15 & 100 \\
\hline Testes Psicológicos & 12 & 80 \\
\hline Entrevistas Coletivas & 8 & 53 \\
\hline Inventários de Personalidade & 5 & 33 \\
\hline Técnicas Projetivas & 5 & 33 \\
\hline
\end{tabular}

micas de grupo, inventários de personalidade, técnicas projetivas e testes psicológicos. Os dados foram organizados em duas tabelas, uma referente às respostas dos psicólogos (Tabela 6) e outra referente às respostas dos estudantes (Tabela $7)$.

Analisando a Tabela 6, constatou-se pela frequiência de respostas que $100 \%$ $(\mathrm{F}=15)$ dos psicólogos utilizam entrevistas individuais e dinâmicas de grupo, 80\% $(\mathrm{F}=12)$ testes psicológicos, $53 \%(\mathrm{~F}=8)$ entrevistas coletivas e $33 \%(\mathrm{~F}=5)$ inventários de personalidade e técnicas projetivas. Foram as entrevistas individuais, as dinâmicas de grupo e os testes psicológicos que apareceram como mais usados pelos profissionais, representando as três técnicas mais freqüentes em seleção profissional.

No que se refere à utilização das técnicas pelos estudantes de Psicologia, verificou-se também pela frequiência de respostas na Tabela 7 que 100\% $(\mathrm{F}=15)$

Tabela 7: Técnicas utilizadas em seleção pelos estudantes de psicologia

\begin{tabular}{|l|c|c|}
\hline \multirow{2}{*}{ Técnicas de Seleção Profissional } & \multicolumn{2}{|c|}{ Técnicas Utilizadas } \\
\cline { 2 - 3 } & F & $\%$ \\
\hline Entrevistas Individuais & 15 & 100 \\
\hline Dinâmicas de Grupo & 14 & 93 \\
\hline Testes Psicológicos & 13 & 87 \\
\hline Inventários de Personalidade & 10 & 67 \\
\hline Entrevistas Coletivas & 10 & 67 \\
\hline Técnicas Projetivas & 7 & 47 \\
\hline
\end{tabular}

usam entrevistas individuais, 93\% ( $\mathrm{F}=14)$ dinâmicas de grupo, 87\% $(\mathrm{F}=13)$ testes psicológicos, $67 \%(\mathrm{~F}=10)$ inventários de personalidade e entrevistas coletivas e $47 \%(\mathrm{~F}=7)$ técnicas projetivas. As três técnicas mais usadas pelos estudantes nos processos seletivos são as mesmas utilizadas pelos profissionais, ou seja, as entre- 
vistas individuais, as dinâmicas de grupo e os testes psicológicos que apareceram com mais freqüência.

Notou-se que $100 \%$ ( $\mathrm{F}=30)$ dos participantes da pesquisa utilizam entrevistas individuais em sua prática profissional, o que demonstra que independentemente dos aspectos que os diferenciam como formação, abordagem ou forma de atuação, é uma técnica vista pelos psicólogos e pelos estudantes de Psicologia como importante e imprescindível em qualquer seleção profissional.

\section{Discussão e CONCLUSÃo}

Os resultados desta pesquisa embora não devam ser generalizados, permitem uma reflexão a respeito do uso e conhecimento de testes nos processos de seleção profissional. Os achados revelam que, confirmando os dados de Noronha (1999), o uso de testes é restrito a uma parcela de profissionais. Nessa mesma direção, pesquisas mostram que os psicólogos conhecem poucos instrumentos, considerando que a tarefa solicitada ao sujeito referia-se a assinalar os nomes dos instrumentos que conheciam/utilizavam, sem ter de fazer qualquer apreciação mais profunda sobre eles (OLIVEIRA; NORONHA; DANTAS, 2005).

A pequena utilização e o desconhecimento dos profissionais acerca dos testes se justifica em razão de variáveis diferentes, tais como a formação profissional, a baixa qualidade psicométrica de muitos instrumentos, a ausência de estudos de validade para os diferentes contextos profissionais, o alto custo de alguns materiais, dentre outros elementos não menos relevantes como já evidenciado pelos pesquisadores (AZEVEDO; ALMEIDA; PAQUALI; VEIGA, 1996; ALMEIDA; PRIETO; MUNIZ; BARTRAM, 1998; NORONHA, 2002; NORONHA; ALCHIERI, 2004).

No que se refere à formação em avaliação psicológica, parecem pertinentes os apontamentos de Alves, Alchieri, e Marques (2002) a fim de que os profissionais saiam mais capacitados, de que haja atualização sistemática dos instrumentos e das técnicas, assim como o planejamento e o desenvolvimento de novos e mais específicos instrumentos. Também sob esta perspectiva, Noronha (2002) enfatiza que os avanços na área apenas serão obtidos à medida que houver incentivo à pesquisa e à avaliação psicológica como um todo ao longo da graduação de forma que as disciplinas ligadas à avaliação e medida psicológica não sejam vistas pelos alunos de forma fragmentada.

Em relação às técnicas utilizadas em seleção profissional, vale destacar que certa incoerência foi encontrada no uso de algumas delas, como já destacado na seção de resultados, assim como os instrumentos de inteligência ao lado dos de personalidade são os mais utilizados. A justificativa para tais achados centra-se no fato de no Brasil haver um maior número de testes que visem avaliar esses construtos ao mesmo tempo em que não estão disponíveis instrumentos padronizados de avaliação para determinados fenômenos psicológicos (NORONHA; VENDRAMINI, 2003).

Essa pesquisa evidenciou, ainda, que as categorias de respostas que apresentaram maior freqüência foram as entrevistas individuais, as dinâmicas de gru- 
po e os testes psicológicos, nessa ordem, como método nos processos seletivos. Nota-se que atualmente esse processo tem passado por profundas transformações, pois, segundo Bodstein (2003) os currículos apresentados nas seleções, que eram a principal preocupação do candidato, hoje são utilizados apenas como roteiro da vida acadêmica e profissional do entrevistado.

Nesse contexto, há evidências de que os selecionadores estão preocupados em contratar um profissional que atenda ao perfil da vaga e que se desenvolva de acordo com a cultura da empresa, adaptando-se às exigências da organização, embora a área organizacional ainda venha enfrentando dificuldades no que se refere à busca do nível de excelência da atividade profissional. Verificou-se que a totalidade dos participantes desse estudo utilizam entrevista individual em sua prática, tendo como segunda opção as dinâmicas de grupo e em seguida, os testes psicológicos. A colocação de Benucci (2003) merece destaque. Ele afirma que não é só a entrevista que conta para o preenchimento de uma vaga, mas também as dinâmicas de grupo e os testes psicológicos, uma vez que contribuem com o índice de acerto na contratação dos candidatos.

Cabe destacar ainda que a aplicação de testes psicológicos é uma das ferramentas mais importantes e necessárias num processo de seleção, pois ajudam o selecionador a traçar um perfil mais preciso do candidato. Cansian (2002) afirma que os testes fornecem aos selecionadores informações dificilmente detectadas em uma entrevista individual ou em uma dinâmica de grupo, mas que, ao mesmo tempo, gradativamente, estão sendo substituídos por essas outras ferramentas que qualquer profissional que trabalhe em seleção de recursos humanos pode utilizar. Detectou-se por meio dessa pesquisa que tal substituição é real, uma vez que a maioria dos sujeitos utiliza mais as entrevistas individuais e as dinâmicas de grupo do que os testes psicológicos em si.

Parece estar claro que os instrumentos utilizados em seleção profissional, mais especificamente os testes psicológicos, necessitam de uma especial atenção no que diz respeito à qualidade, manuseio e formação dos profissionais que os utilizam. Noronha (2002) ressalta que essa é uma discussão que envolve muitas instâncias do ser e do fazer psicológico. Por um lado, existe o psicólogo que atua na prática, mas que não está atualizado, por outro, existem instituições formadoras que não estão formando de acordo com as necessidades do profissional, e, por outro lado ainda, tem-se o conhecimento de pesquisadores, nem sempre transmitidos forma eficaz aos psicólogos que estão nas práticas profissionais.

Portanto, sugere-se que ações para avaliação psicológica sejam estudadas, com objetivo de aprimorar os instrumentos e as técnicas de acordo com as reais necessidades das empresas, desenvolver novos instrumentos, melhorar a formação profissional na graduação, bem como revisar os currículos dos cursos de Psicologia, uma vez que o ensino de avaliação psicológica encontra-se, algumas vezes, 
defasado. Embora os avanços da avaliação sejam claros, detectou-se a importância de se continuar fazendo estudos e pesquisas na área com o intuito de que profissionais de recursos humanos atuem com maior eficácia na seleção profissional.

\section{REFERÊNCIAS}

ALCHIERI, J. C. Critérios de escolha dos Instrumentos Psicológicos. data. Disponível em: <http://www.saúde.unisinos.br> Acesso em: 2003.

ALCHIERI, J. C. Dilemas do psicotécnico: exame psicotécnico ou avaliação psicológica? In: CONGRESSO NACIONAL DE AVALIAÇÃO PSICOLÓGICA, 8., 1999, Porto Alegre. Anais...Porto Alegre: [s. n.], 1999. p. 208-215.

ALMEIDA, L. S., et al. D. O uso dos testes em Portugal, Espanha e Países Iberoamericanos. Psychologica, local, n. 20, p. 41-55, 1998.

ALVES, I. C. B.; ALCHIERI, J. C.; MARQUES, K. C. As técnicas de exame psicológico ensinadas nos cursos de graduação de acordo com os professores. Psico-USF, local, v. 7, n. 1, p. 77-88, 2002.

AZEVEDO, M. M., et al. Utilização dos Testes Psicológicos no Brasil: dados de estudo preliminar em Brasília. In: ALMEIDA, L. S. et al. Avaliação psicológica: formas e contexto. Braga: [s. n.], 1996.

BASTOS, A. V. B. Perfis de formação e ênfases curriculares: o que são e por que surgiram? Revista do Departamento de Psicologia - UFF, Niterói, v. 14, n. 1, p. 31-58, 2002.

BENUCCI, M. Entrevista e processo seletivo. Data. Disponível em:http:// www.rh.com.br/artigos. Acesso em: 2003.

BODSTEIN, L. R. O mercado de trabalho na era da globalização. Data. Disponível em: 〈http://www.rh.com.br/artigos>. Acesso em: 2003.

CANSIAN, R. M. M. O psicólogo e seu espaço nas organizações. data. Disponível em: <http://www.cjh.ufsc.br/sinapsi/artigos/organizacional3htm>. Acesso em: 2002 .

CASTELAR, M. Mercado. Jornal de Psicologia, São Paulo, edição 115, 2001.

DURAN, A. P. Alguns dilemas na formação do psicólogo: buscando sugestões para superá-los. In Psicólogo Brasileiro: práticas emergentes e desafios para a formação. São Paulo: Casa do Psicólogo,1994, p. 45-53.

FIGUEIREDO, V. L. M.; PINHEIRO, S. O Teste WISC-III em uma amostra do Rio Grande do Sul. Temas em Psicologia, Porto Alegre, v. 6, n. 3, p. 255-261, 1998. 
HUTZ, C. S.; BANDEIRA, D. R. Avaliação psicológica no Brasil: situação atual e desafios para o futuro. In: YAMAMOTO, M. A. H.; GOUVEIA, V. V. (Org.). Construindo a psicologia brasileira: desafios da ciência e prática psicológica. São Paulo: Casa do Psicólogo, 2003.

NORONHA, A. P. P. et al. Em defesa da avaliação psicológica. Data. Disponível em: <http://www.ibapnet.org.br/manifesto.asp >. Acesso em: 2003.

NORONHA, A. P. P. Os problemas mais graves e mais freqüentes no uso dos testes psicológicos. Psicologia: Reflexão e Crítica, Porto Alegre, v. 15, n. 1, p. 135-142, 2002.

NORONHA, A. P. P. Avaliação psicológica segundo psicólogos. 1999. Tese (Doutorado)-Instituto de Psicologia, Pontifícia Universidade Católica de Campinas, Campinas, SP, 1999.

OLIVEIRA, K. L.; NORONHA, A. P. P.; DANTAS, M. A. O psicólogo comportamental e o uso de técnicas e instrumentos psicológicos. Psicologia em Estudo, Maringá, v. 10, n. 1, p. 127-135, 2005.

PEREIRA, F. M.; PRIMI, R.; COBÊRO, C. Validade de testes utilizados em seleção de pessoal segundo recrutadores. Psicologia: Teoria e Prática, São Paulo, v. 5, n. 2, p. 83-98, 2003.

PRIETO, G.; MUÑIZ, J. Um modelo para evaluar la calidad de los tests utilizados em España. Disponível em: 〈http://www.cop.es/tests/modelo.htm>. Acesso em: 2000.

SCHIMDT , F. L.; HUNTER, I. E. The validity and utility of selection methods in personnel psychology: practical and theoretical implications of 85 years of research findings. Psychology Bulletin, v. 124, no. 2, p. 23-38, 1998.

Recebido em: setembro/2004 Aceito em: março /2005 
